\title{
Autonomous Transport Mean Reverse Movement Control by Nodal Points
}

\author{
Michael Rachkov*, Sergey Petukhov \\ Department of Mechanical Engineering, Moscow Polytech, Moscow, Russia \\ Email address: \\ michyur@gmail.com (M. Rachkov) \\ ${ }^{*}$ Corresponding author
}

\section{To cite this article:}

Michael Rachkov, Sergey Petukhov. Autonomous Transport Mean Reverse Movement Control by Nodal Points. American Journal of Engineering and Technology Management. Vol. 4, No. 5, 2019, pp. 73-78. doi: 10.11648/j.ajetm.20190405.11

Received: September 20, 2019; Accepted: September 24, 2019; Published: November 22, 2019

\begin{abstract}
The study presents an exact formulation of control for the reverse moving a transport mean along a polygonal course consisting of rectilinear segments interconnected by nodal points. Appropriateness of the question is caused by the need to figure out a several of tasks: to secure the transport mean in the occasion of a communication crash by returning along the course already passed, to escape rotation in constrained or unsafe conditions, or partial go back for the following avoid of the hurdle and continuation of the forward motion. The control method of forward motion assumes that the route of movement is predetermined, and the path is elaborated by using landmarks. Video cameras are placed on the transport mean for landmark measurement. They are controlled by the operator through the radio channel. Errors in estimating deviation from the supposed course are detected using the multidimensional correlation investigation instrument based on the dynamics of a lateral deviation mistake and a velocity mistake. Reception algorithm of the information is presented on reference points. The outcome of the test showed a considerable preciseness in determining the position vector that provides the reverse movement relative to the reference course with a reasonably admissible mistake while returning to the start spot.
\end{abstract}

Keywords: Automatic Control, Reverse Motion, Navigation, Landmarks, Correlation Analysis, Reference Trajectory

\section{Introduction}

The improvement of systems for self-acting return based on the trajectories measured and stored in the on-board computer corresponds to the common tendency of the development of autonomous and remotely operated transport means. Among such may be planet-walkers, mobile robots for movement in constrained conditions, robotic combat vehicles, etc. It is assumed that the data about landmarks are obtained by means of a stereo television system on board the vehicle. The solving of the question is connected with the use of adaptive control and intellectual data processing.

Applicability of the question is caused by the need to resolve several tasks, namely, to preserve the transport mean in the event of communication crash by returning along the course already passed, to escape rotation on the area in constrained or hazardous conditions (demining), or fragmentary back movement for avoiding the obstacle and continuing the advance motion $[1,2]$. Settlement of transport mean course parameters and a position of the transport mean by navigation measurements, computation of course corrections and improvement of control system settings for the upcoming maneuvers constitute the core of the navigational mission [3-12]. The camera use for navigating movable systems has been labored for different applications [13-15]. Map representation for robotic systems is effective in some tasks [16]. The object of the paper research is improvement of these studies in the way of solving the question of reverse moving optimization by using the passed course data.

\section{Formulation of the Problem}

The control task of automatic return of the transport mean on memorized landmarks provides two stages of transport mean movement:

1) Forward motion with saving trajectory parameters and a layout of the landmarks $P$, the length of the travel path $l$, the angle $\varphi$ of course rotation and the rotation angle 
of the stereo camera $\beta$;

2) Self-acting come back by reverse movement with the help of the stored mark plans and newly obtained point plans for corrections of the course.

It is assumed that the transport mean is moving along a multilink polygonal trajectory consisting of rectilinear lengths $l_{k}$ connected by nodal points $S_{k}, k=1,2, \ldots, N-1$. Such a course requires a reduced number of parameters and the amount of memory to stock in the on-board computer. Landmark measurements are made at some points, called reference points (Figure 1)

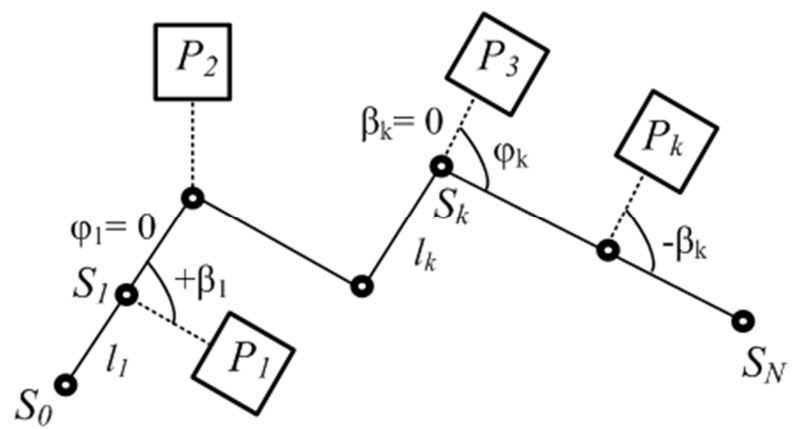

Figure 1. Diagram of binding the nominal trajectory to the plans of the neighborhoods $P_{k}$.

The vector of steering measurements $M_{k}$ in the $k$-th reference point can commonly be written in the following way:

$$
M_{k}=\operatorname{col}\left(l_{k}, \varphi_{k}, \beta_{k}, P_{k}\right)
$$

If the valuation is not made at the node then the measuring vector is undeveloped: $M_{k}=\operatorname{col}\left(l_{k}, \varphi_{k}, 0,0\right)$. The course passed and stored in the system of tele-controlled forward movement into the working tract is nominal for self-acting go back of the transport mean.

The method of navigation with forward motion assumes that a path of the motion is prearranged, and the route is precise provided that it passes near the landmarks. Cameras are placed on the transport mean controlled by the operator with the help of a wireless channel. They measure landmarks while moving. The set-up of point plans takes place in the following progression.

1) The operator searches for landmarks by turning on the angle $\beta_{k}$ the pointing device of the stereo unit during the stop.

2) Stereo images go into the on-board computer and are processed.

3) A compressed image of landmarks on the reference surface of moving is formed by projections of vertical lines in the configuration of point plans $P_{k}$ in a coordinate system associated with the transport mean.

4) The parameters $\left(l_{k}, \varphi_{k}, \beta_{k}\right)$ and the resulting point plans $P_{k}$ are saved for the self-acting go back of the transport mean.
5) The next part of the course at each node $S_{k}$ is put by the settings $\left(\varphi_{k}, l_{k+1}\right)$, which are transmitted by the operator to the board.

The method for navigating an autonomous transport mean when moving to a work area requires parameters $\left(\tilde{l}_{k}, \tilde{\varphi}_{k}, \tilde{\beta}_{k}\right)$, where $k=1, \ldots, N-1$. A pre-compiled placement map stored in the on-board computer memory in the configuration of a progression of point plans $\tilde{P}_{k}$ is used for steering at reference points. Wherein:

1) Landmarks are captured in the survey area of cameras at the point $S_{k}$ by turning the stereo system to the angle $\tilde{\beta}_{k}$;

2) The course improvement is performed on the base of the coordination results of the plans $\tilde{P}_{k}$ and the newly obtained point plans $P_{k}$ that are stored for self-acting go back;

3) Studied values $l_{k}, \varphi_{k}, \beta_{k}$, and point plans $P_{k}$ of landmarks are used for self-acting go back.

The grounds of autonomous navigation in self-acting back movement is based on the use of the operational machine relative map (MRM), automatically formed in the process of direct motion of the transport mean at the nodal and reference points. Such information is represented in the memory of the on-board computer in the arrangement of a stack. Each "page" of the MRM corresponding to the reference point $k$ contains parameters $\left(l_{k}, \varphi_{k}, \beta_{k}\right)$ along with a point plan $P_{k}$. If there are no reference points in the nodal points, then the corresponding "page" of the MRM is represented only by parameters $\varphi_{k}, l_{k}$. In the reverse movement, the MRM parameters are extracted from the computer memory stack in the reverse arrangement, in accordance with the finish point number of the transport mean. In the MRM, the point plans are linked through the parameters of the course and shape a singular knowledge area.

The parameters $\varphi_{k}, l_{k}$ of the course are worked out by the control system with errors, so there is a deviation of the moving from the planned point $S_{k-1}$ to the point $S_{k}^{\prime}$ at which the stereoscopic survey is performed and the point plan $P_{k}^{\prime}$ is obtained. As an outcome of the alignment of the plan $P_{k}^{\prime}$ with the plan $P_{k}$, the deviations from the trajectory $\left(\Delta \bar{r}_{k}, \Delta \varphi_{k}\right), \Delta \varphi_{k}=\varphi_{k}^{\prime}-\varphi_{k}$ and the command parameters $\left(\hat{r}_{k}, \hat{\varphi}_{k}\right)$ that assure the transport mean movement to the point $S_{k-1}$ are identified (Figure 2). 


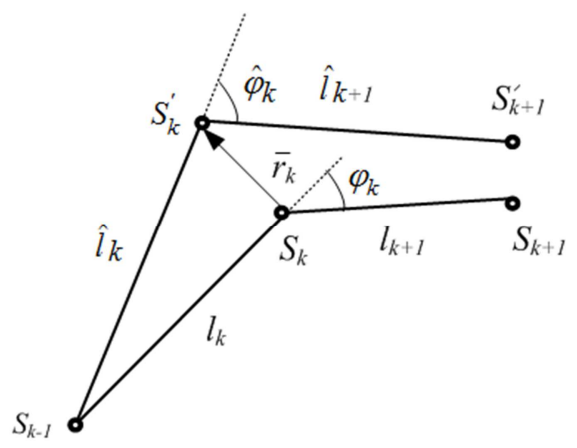

Figure 2. Diagram for determining path length $\hat{l}_{k}$ and heading angle $\hat{\varphi}_{k}$.

The main task is settlement of the benchmark plans for calculating the navigation parameters $\left(\bar{r}_{k}, \Theta_{k}\right)$ shown in Figure 2, and the control parameters $\left(\hat{l}_{k}, \hat{\varphi}_{k}\right)$ to proceed with moving to the next node. Diagram of the reverse motion course is shown in Figure 3.

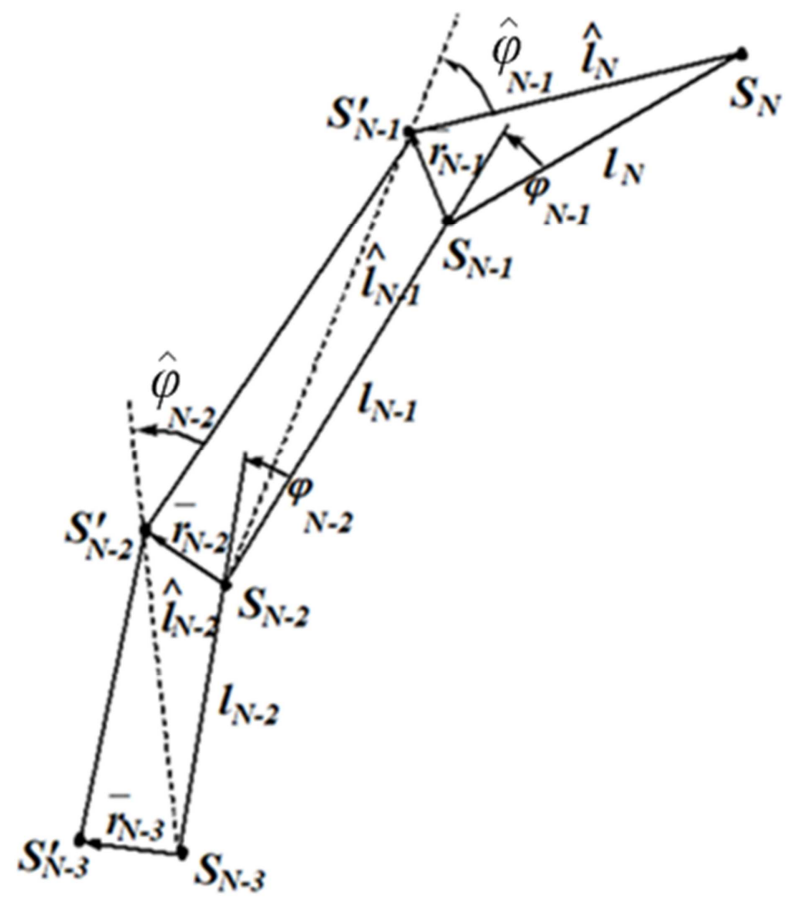

Figure 3. Diagram of the reverse movement trajectory.

Mathematical statement of control tasks with automatic return is the same for remote control and autonomous movement. Parameters $l_{k}, \varphi_{k}, \beta_{k}$ and $P_{k}$ have random character, therefore the nominal course is probabilistic, as well as the go back track.

\section{Calculation of Motion Parameters}

The movement representation in the locality of the stored course is based on the picture of the disturbed movement relative to the planned course by a system of linear differential equations. It is supposed that the transport mean moves rectilinearly between the nodal points at a fixed velocity. The most significant mistake component is caused by the constantly increasing lateral deviation due to the mistake in the course, which leads to deviations from the goal point $\delta x_{k} \approx V \cdot T_{k+1} \cdot \delta \varphi_{k}$, where $T_{k+1}$ - duration of the motion from point $\mathrm{Sk}+1$ to point $S_{k}$. Both quantities $\delta l$ and $\delta \varphi$ are random and influence the deviation from the point, which is supposed to be subordinate to the normal distribution law.

Errors in estimating deviations from the titular course of movement are resolved using the multidimensional correlation analysis instrument based on the dynamics of the mistake changing of the lateral deviation $\rho_{1}$ and the transport mean velocity $\rho_{2}$ of deviation from the titular course. The values $\rho_{1}$ and $\rho_{2}$ are components of the state vector $\bar{\rho}$. Differential equations of perturbed movement in matrix configuration are the next:

$$
\frac{d \bar{\rho}}{d t}=A \bar{\rho}, \quad \bar{\rho}=\left|\begin{array}{l}
\rho_{1} \\
\rho_{2}
\end{array}\right|, \quad A=\left|\begin{array}{ll}
0 & 1 \\
0 & 0
\end{array}\right|,
$$

where $A$ is the linear differential equations system matrix,

$$
\rho_{1}=\delta x_{k}, \quad \rho_{2}=d \rho_{1} / d t=V \cdot \delta \varphi_{k},
$$

with the initial conditions

$$
\rho_{1}(0) \in(0, \delta r), \rho_{2}(0) \in(0, V \cdot \delta \theta)
$$

and a correlation matrix of initial deviations

$$
\Sigma_{\rho}=\left|\begin{array}{ll}
\sigma_{1}^{2} & K_{12} \\
K_{21} & \sigma_{2}^{2}
\end{array}\right|, \quad K_{12}=K_{21}=K \sigma_{1} \sigma_{2},
$$

where $\sigma_{1}$ and $\sigma_{2}$ are the mistake variances in the determination of $\rho_{1}$ and $\rho_{2} ; K$ - a correlation coefficient.

The state of the system $\bar{\rho}(T)$ at the terminal moment of time $T$ is set by means of a transition matrix $\Phi(T, t), t \in(0, T)$, that is identified from the solution of the conjugated system [6]. With the help of this transition matrix, the system state vector is ascertained at the terminating moment of time $T$ according to the situation at the current instant:

$$
\bar{\rho}(T)=\Phi(T, t) \bar{\rho}(t) ; \rho_{1}(T)=\Phi_{1}(T, 0) \bar{\rho}(0),
$$

where $\Phi_{1}(T, 0)$ is the transition matrix first row.

The utmost possible deviation from the node $S_{k}$ due to a position inaccuracy $\delta r$ and a mistake in the implementation of the rotation angle $\delta \varphi$ is obtained by substituting the value $T=\hat{T}$ of the predicted movement time from point $S_{k+1}$ to point $S_{k}$ and the extreme possible deviations in the components of the state vector $\bar{\rho}(0)$ at the start instant: 


$$
\rho_{1}\left(\hat{T}_{i}\right)=\left|\begin{array}{cc}
1 & \hat{T}
\end{array}\right| \begin{gathered}
\delta r \\
3 V \sigma_{\varphi}
\end{gathered} \mid=\delta r+3 V \hat{T} \sigma_{\varphi}
$$

The predicted value of the discrepancy of deviation from the designed course at the moment $\hat{T}$ relatively the start time $(t=0)$ is:

$$
\sigma_{x}^{2}=\Phi_{1}(\hat{T}, 0) \Sigma_{\rho} \Phi_{1}^{*}(\hat{T}, 0)
$$

where $(*)$ is the transpose sign, and $K$ is the correlation coefficient. The correlation coefficient determines the placement of the dispersion ellipse.

The goal of the improvement is to limit the deviation $r_{0}$ from the starting $\operatorname{dot} S_{0}$, but at the same time the ongoing deviations $r_{k}$ from the titular course, controlled at the reference points, also should not surpass the allowable value: $r_{k} \leq R_{d}$, where $k=1,2, \ldots, N-1$, and $R_{d}$ is the utmost allowable deviation. This circumstances, in combining to safeness considerations while returning, involves taking camera pictures from closely spaced points to the titular course and minimizing the differences in the plan of landmarks $P_{k}^{\prime}$ from the plan $P_{k}$. The span of the possible go back paths is determined at the node points relative to the nominal course by dispersion of ellipses $R_{k}, k=N-1, \ldots, 0$.

\section{Reception of the Information on Reference Points}

Dot plans are formed by measurement of reference points with participation of the operator and in an automatic operating mode by means of the stereo camera system. The first way has a high degree of reliability due to an opportunity of the operator to observe reference points and obstacles on the screen. The point plan turns out to be free from interference and represents a point plan of landmarks. It is an invariable configuration of points, the shape and dimensions of which are invariant to the direction and range of measuring (Figure 4).

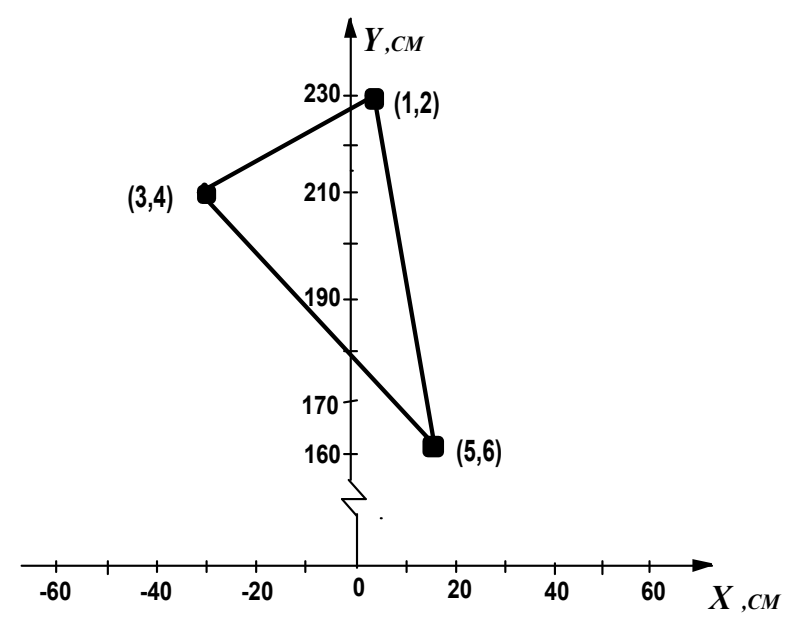

Figure 4. The dot plan of reference points in a projection to the motion surface (the measured points are numbered from 1 up to 6).

Coordinates of measured points are $X$ (lateral coordinate), $Y$ (range), and $Z$ (height). Elements of the dot plan of reference points are designated by numbers of the corresponding measured points. Linear displacement of dot plans on position $\bar{r}$ and their relative corner of turn $\Theta$ are taken into account at different capacity of the sets representing dot plans.

Let's enter systems of coordinates $x_{k} S_{k} y_{k}$ and $x_{k}^{\prime} S_{k}^{\prime} y_{k}^{\prime}$ into planes of movement of the robot (Figure 2) with axes $S_{k} y_{k}$ and $S_{k}^{\prime} y_{k}^{\prime}$ combined with corresponding pieces of trajectories: memorized and realized at backward movement. Mutual position of dot plans is defined by affine transformation, including turn on a corner $\Theta$, carry $\bar{r}$ and change of scale $\mu$ :

$$
\bar{R}_{P}=\mu M(\Theta) \bar{R}_{P^{\prime}}+\bar{r},
$$

where $\bar{r}=(\Delta x, \Delta y)^{T}$ is a vector of displacement of the plan $P^{\prime}$ in the coordinate system of the plan $P$ equal to a vector of a deviation of the transport mean from a corresponding reference point; $\bar{R}_{P}, \bar{R}_{P^{\prime}}$ are radiuses-vectors of the center of reference points gravity in coordinate systems of plans $P$ and $P^{\prime} ; M(\Theta)$ is a cosine guide matrix determined by turn of the plan $P^{\prime}$ relatively the plan $P$. In our case, the scale of the dot plan of reference points is constant, as reference points are rigid, therefore $\mu=1$.

The solution of information reception task by results of the coordination of dot plans is shown in Figure 5 . Mathematically, the problem is reduced to finding the limit of the sequence of projections onto convex sets, which is represented by the intersection of two point sets of different powers. When using the stereo camera, the task is practically reduced to the classical task of recognition by reference.

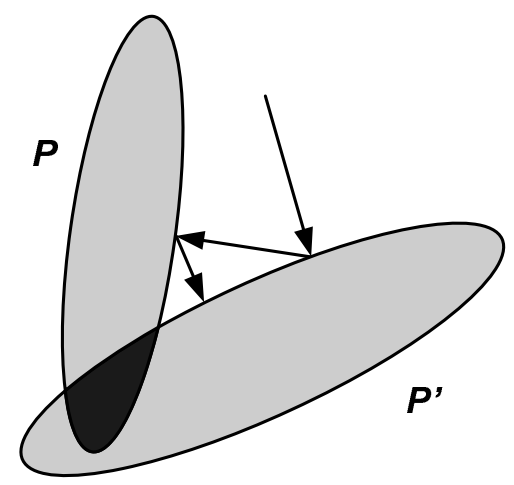

Figure 5. Limit of the sequence of projections onto convex sets.

Representation of reference point plans significantly increases the speed and reliability of matching algorithms. Additional acceleration is obtained by using the "coloring" of each element of the point plan with a parameter $(\Pi= \pm 1)$ that is a sign of the intensity gradient in vertical reference points.

In order to increase the reliability of solving the problem, additional features are introduced. There are invariants built 
on points from sets $M_{P}$ and $M_{P^{\prime}}$ not changing their configuration when moving from plan $P$ to plan. $P^{\prime}$. Such can be considered ruled segments of the same line of the same name, connecting identically colored points on the plans of landmarks, and (or) the morphological signs formed by them as geometric figures (triangles). The fast matching algorithm for point plans is based on sequential enumeration of line invariants or line segments connecting pairs of points on the plans. The essence of the algorithm is that for the first iteration of all possible segments of the plan $P$ and $P^{\prime}$ satisfy the condition

$$
\left|d_{P}-d_{P^{\prime}}\right|<0.01 d_{P}
$$

where $d_{P}, d_{P^{\prime}}$ are the lengths of the segments on the plans and which are on the plans due to the invariance of the geometric configuration of the reference points. The implementation of the algorithm is as follows.

1) For each pair of segments, the mismatch angle $\Delta \Psi_{i}$ is determined, and the average value at the $\mathrm{n}$-th step is

$$
\Delta x=x_{P}+\left(y_{P^{\prime}} \sin \Theta-x_{P^{\prime}} \cos \Theta\right),
$$

where $\left(x_{P}, y_{P}\right)$ and $\left(x_{P^{\prime}}, y_{P^{\prime}}\right)$ are coordinates of a point $\mathrm{C}$ on dot plans $P$ and $P^{\prime}$.

\section{Experimental Results}

As an experimental example, the obtained interval control was tested for a mobile wheel robot. The necessary condition is the movement of the robot from each current node $S_{k}$ to the next node $S_{k-1}$. Control parameters are selected from the approximation conditions near node points.

The robot hardware included an on-board computer. The passed distance was measured by a sensor of driving wheel speed. Rotation of the robot was performed by rotating left and right wheels in opposite directions. The system software consisted of programs for automatic analysis of stereo images, programs for formation and coordination of point plans, and calculation of the guidance parameters $\left(\hat{r}_{k}, \hat{\varphi}_{k}\right)$ in the vicinity of the $k$-th reference point. The robot motion trajectory in the room along 6 reference points and 4 points of rotation and the trajectory of reverse motion to the initial point are shown in Figure 6. calculated as the functional:

$$
\Delta \Psi_{n}=\frac{1}{M} \sum_{i=1}^{M} \Delta \Psi_{i},
$$

where $M$ is the number of remaining invariants on the plans $P$ and $P^{\prime}$.

2) The least "similar" segments along the length and angle of inclination are excluded, the value of the functional is compared with its value in the previous step, and paragraph $1)$ is repeated.

3) Coordination of point plans continues until the minimum value of the functional is obtained or when $M_{P}=$ $M_{P^{\prime}}=2$. In this case, the center of gravity $C$ of the common part of the point plans $P, P^{\prime}$ and their relative rotation angle $\Theta=\Delta \Psi_{n}$ are calculated.

According to the results of the coordination of point plans, the projections of the relative displacement vector are determined in accordance with the transformation

$$
\Delta y=y_{P}+\left(y_{P^{\prime}} \cos \Theta+x_{P^{\prime}} \sin \Theta\right)
$$

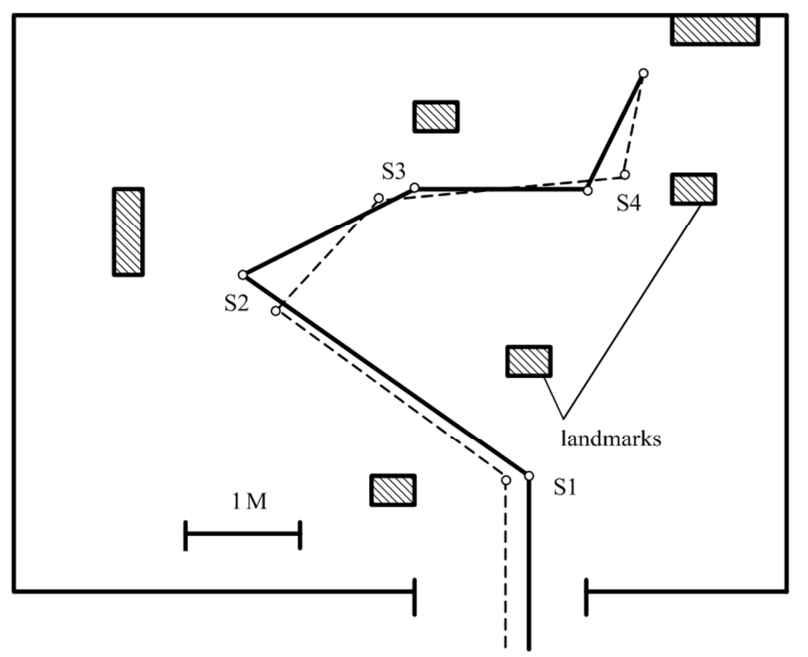

Figure 6. Trajectories of robot motion in the room (solid line-direct motion, dashed line-reverse motion).

The outcome of the test showed adequate exactness of determining the state vector $\left(r_{N-k}, \Delta \hat{l}_{N-k}, \hat{\varphi}_{N-k}\right)$ according to the results of the point plans corresponding, providing reverse motion relative to the reference trajectory with an satisfactory inaccuracy about $0.2 \mathrm{~m}$ at the start point. The algorithm has high performance. The cost of computer time is hundredths of a second.

\section{Conclusion}

A precise formulation of the transport mean reverse movement along a multi-link polygonal course consisting of rectilinear segments interconnected by nodal points is determined. The control method of navigation with direct movement assumes that the route of movement is measured, 
and the path is corrected by using landmarks. A block of cameras controlled by the operator through the radio channel is placed on the transport mean to measure landmarks on board.

Errors in deviation estimating from the titular course of movement are resolute using the multidimensional correlation analysis instrument based on the dynamics of the lateral deviation mistake and velocity mistake of the transport mean.

The idea of autonomous navigation in self-acting reverse motion is based on the use of an operational machine relative map automatically formed during the forward motion of the transport mean at the node and reference points. The transport mean movement model in the neighborhood of the stored course is based on the depiction of the disturbed moving relative to the planned course by a system of linear differential equations. It is supposed that the transport mean moves rectilinearly at a fixed velocity between the nodal points.

The outcome of the test showed a relatively high accuracy in determining the state vector that provides a reverse movement accordingly to the reference trajectory with a practically satisfactory position error while transport mean returning to the start point.

\section{References}

[1] L. Thrybom, J. Neander, E. Hansen, and K. Landernas. Future challenges of positioning in underground mines. In Proceedings of the IFAC Conference on Embedded Systems, Computer Intelligence and Telematics, pp. 222-226, June 2015.

[2] T. Nothdurft, P. Hecker, S. Ohl, F. Saust, M. Maurer, A. Reschka, and J. Bohmer. Stadtpilot: First fully autonomous test drives in urban traffic. In Proceedings of the IEEE/RSJ International Conference on Intelligent Robots and Systems, pp. 919-924, October 2011.

[3] S. Petukhov, M. Rachkov. Navigation Method of Autonomous Robot Backward Motion by Remembered Landmarks // 12th Int. Conf. on Climbing and Walking Robots and the Support Technologies for Mobile Machines. - 2009. - pp. 19-25.

[4] M. Rachkov, S. Petukhov, Navigation of the autonomous vehicle reverse movement, Journal of Physics: Conference Series, V. $315 \quad$ (2018) 012019 doi: 10.1088/1757899X/315/1/012019, pp. 1-7, 2019.

[5] S. Lefèvre, D. Vasquez, and C. Laugier. A survey on motion prediction and risk assessment for intelligent vehicles. Robomech Journal, 1 (1): 1, 2014.
[6] R. Attia, R. Orjuela, and M. Basset. Combined longitudinal and lateral control for automated vehicle guidance. Vehicle System Dynamics, 52 (2): 261-279, 2014.

[7] J. Kong, M. Pfeiffer, G. Schildbach, and F. Borrelli. Kinematic and dynamic vehicle models for autonomous driving control design. In Proceedings of the IEEE Intelligent Vehicles Symposium, pp. 1094-1099, June 2015.

[8] V. Turri, A. Carvalho, E. Tseng, K. Johansson, and F. Borrelli. Linear model predictive control for lane keeping and obstacle avoidance on low curvature roads. Proceedings of the International IEEE Intelligent Transportation Systems Conference, pp. 378-383, October 2013.

[9] C. Beal and J. Gerdes. Model predictive control for vehicle stabilization at the limits of handling. IEEE Transactions on Control Systems Technology, 21 (4): 1258-1269, 2013.

[10] A. Carvalho, Y. Gao, S. Lefevre, and F. Borrelli. Stochastic predictive control of autonomous vehicles in uncertain environments. Proceedings of the International Symposium on Advanced Vehicle Control, September 2014.

[11] F. Borrelli, A. Bemporad, and M. Morari. Predictive control for linear and hybrid systems. 2015. $440 \mathrm{p}$.

[12] DeSouza G. N., Kak A. S. Vision for Mobile Robot Navigation // IEEE Trans. on PAMI. - 2002. - № 24. - P. 237 267.

[13] Petukhov S. V., Ivaniugin V. M. Interaction with external environment of robot by man-machine interface // Proceedings of the 2nd Inter. Conf. On Climbing and Walking Robots, 1999. - pp. 445-452.

[14] Petuhov A. S., Rachkov M. Ju., Petuhov S. V. Application of compressed image post-processing algorithms for remote control of mobile robots // Mechatronics, automation, control. - 2007. - №1. - pp. 17-24 (in Russian).

[15] Measuring systems of a robotic complex for operation in a nuclear reactor / Gradeckij V. B., Rachkov M. Ju., Petuhov S. V., et al. - Preprint № 656. - Russian Academy of Sciences, 1999. - 46 p. (in Russian).

[16] K. Wurm, A. Hornung, M. Bennewitz, C. Stachniss, and W. Burgard. Octomap: A probabilistic, flexible, and compact 3D map representation for robotic systems. In Proceedings of the IEEE Conference on Robotics and Automation, vol. 2, May 2010. 